\title{
Atorvastatin inhibits collar-induced intimal thickening of rat carotid artery: Effect on C-type natriuretic peptide expression
}

\author{
YONGJIE LI, YUAN GAO and DINGYIN ZENG
}

\author{
Department of Cardiology, The First Affiliated Hospital of China Medical University, \\ Shenyang 110001, P.R. China
}

Received October 12, 2011; Accepted December 1, 2011

DOI: $10.3892 / \mathrm{mmr} .2011 .698$

\begin{abstract}
The present study was performed to elucidate the mechanism underlying the anti-atherogenic action of atorvastatin (ATV). We investigated the effect of ATV on intimal thickening of the rat carotid artery induced by collar placement and further examined the effect of ATV on the expression of C-type natriuretic peptide (CNP), cGMP-dependent protein kinase (PKG) I $\alpha$ and PKG I $\beta$ in carotid arteries. The expression of CNP was examined by enzyme-linked immunosorbent assay (ELISA) and quantitative real-time RT-PCR. Western blotting was used to determine the expression of PKG I $\alpha$ and PKG I $\beta$. After 14 days, the collar placement induced a marked neointima formation and a reduction in CNP and PKG I $\alpha$ expression. These effects were significantly reversed by ATV treatment. However, no obvious changes in PKG I $\beta$ expression were observed throughout the study. In conclusion, the present data suggest that elevation of CNP represents an additional mechanism by which ATV treatment may prevent the development and progression of atherosclerosis.
\end{abstract}

\section{Introduction}

Atherosclerosis, the major cause of cardiovascular disease, is a multifaceted, progressive, inflammatory disease in which the formation and build-up of atherosclerotic plaques cause hardening and narrowing of major arteries. Atherosclerosis may lead to several important adverse vascular events including coronary artery disease (CAD), stroke and peripheral arterial disease, responsible for most of the cardiovascular morbidity and mortality worldwide (1-3). Statins, which are 3-hydroxy-3-methylglutaryl coenzyme A (HMG-CoA) reductase inhibitors, have emerged as effective agents in lowering elevated levels of low-density lipoprotein-cholesterol (LDL-C), one of the principal risk factors for atherosclerosis

Correspondence to: Dr Dingyin Zeng, Department of Cardiology, The First Affiliated Hospital of China Medical University, 155 North Nanjing Street, Shenyang 110001, P.R. China

E-mail: dingyin_zeng@163.com

Key words: atherosclerosis, peri-arterial collar, intimal thickening, atorvastatin, C-type natriuretic peptide, cyclic guanosine monophosphate-dependent protein kinase and its pursuant manifestations (4). Reductions in total LDL-C achieved by statin treatment have been shown to translate into reductions in the risk of cardiovascular morbidity and mortality in both primary and secondary prevention settings (5-8). However, there is increasing evidence for additional benefits of statins that cannot be fully explained by their lipid-lowering effect $(9,10)$. Several clinical trials demonstrated anti-inflammatory and immunomodulatory effects of treatment with statins that would represent the likely explanations for further benefits attributable to this class of drugs (11-13). Nonetheless, the mechanisms underlying the anti-inflammatory effect of statin treatment have not yet been fully elucidated.

C-type natriuretic peptide (CNP), a 22-amino acid peptide, belongs to a family of structurally related cardiovascular hormones also including atrial natriuretic peptide (ANP) and B-type natriuretic peptide (BNP). ANP and BNP are synthesized in cardiomyocytes and released into the circulation in significant amounts, thereby contributing to the regulation of cardiovascular homeostasis. In contrast, CNP is secreted by vascular endothelial cells and acts locally as a regulator of vascular tone and growth through intracellular accumulation of cyclic guanosine monophosphate (cGMP) $(14,15)$. Furthermore, CNP appears to have both anti-inflammatory and anti-mitogenic properties, suggesting that it may also be protective against the development of atherosclerotic lesions $(16,17)$. In support of this, CNP has been reported to inhibit the intimal thickening in a number of experimental models of atherosclerosis, and expression of CNP and its receptors NP receptor (NPR)-B and -C in human coronary arteries are inversely correlated with severity of atherosclerotic lesions (18-21). Additionally, CNP also exerts anti-migratory and anti-proliferative effects on vascular smooth muscle cells (VSMCs) in vitro (15).

Based on these previous studies on statins and CNP, we raised the hypothesis that statins may protect against the development of atherosclerosis, at least partially through the elevation of CNP expression. To test our hypothesis, we examined the effects of atorvastatin (ATV) treatment on intimal thickening and CNP expression in collar-induced atherosclerotic rats.

\section{Materials and methods}

Animal model of atherosclerosis. Male adult rats weighting from 220 to $250 \mathrm{~g}$ were purchased from the Experimental 
Animal Centre of China Medical University and fed a regulatory diet (zero cholesterol) 1 week before surgery. Rats were anesthetized by intraperitoneal injection of chloral hydrate $(300 \mathrm{mg} / \mathrm{kg})$. Subsequently, a midline neck incision was made to surgically expose the right carotid artery, and a non-occlusive silastic collar (length $10 \mathrm{~mm}$, inner diameter $1.5 \mathrm{~mm}$ ) was positioned around the right carotid artery and held in place with a nylon sleeve. The carotid arteries were then returned to their original position and the entry wound was sutured. After recovery from the anesthesia, the animals received their respective treatment for 2 weeks. The rats in the sham group underwent the same procedure except the collar placement. All animal study protocols were approved by the Ethics Committee for Animal Experiments of China Medical University, and the animals received human care in compliance with the Principles of Laboratory Animal Care.

Experimental design. After surgery, rats were divided into two groups receiving ATV at a dose of $10 \mathrm{mg} / \mathrm{kg} / \mathrm{day}$ or vehicle (5\% glucose solution), respectively. The dosage of ATV administered in this study was based on previous studies (22-24). Rats were assigned randomly to each of the treatment groups and received ATV or vehicle for 14 consecutive days by oral gavage. The sham-operated rats received a regulatory diet only. During the study, animals were provided ad libitum with a standard diet and water.

Tissue harvesting and morphometry. At the end of the study, all rats were sacrificed with an overdose of phenobarbital and two segments were cut from the collared and sham-operated artery, one for western blot analysis, the other for morphometry. The former was immediately frozen at $-80^{\circ} \mathrm{C}$ until needed. The latter was immediately placed in formalin fixative solution (4\%) for $24 \mathrm{~h}$, dehydrated in a graded series of isopropyl alcohol (60-100\%) followed by toluol before being embedded in paraffin. Transverse 5- $\mu \mathrm{m}$ tissue sections were cut and routinely stained with hematoxylin and eosin (H\&E). The cross-sectional area of the intima and media in each artery and the ratio of these values were calculated (intima/media area ratio, IMR).

Enzyme-linked immunosorbent assay (ELISA). CNP expression in artery segments was assayed using an ELISA kit (EIAab Science Co., Ltd, Wuhan, China). In brief, total proteins were extracted from artery segments and the protein concentrations were determined by the bicinchoninic acid (BCA) assay. Then, CNP concentrations by ELISA were carried out according to the manufacturer's instructions.

Quantitative real-time RT-PCR. Total-RNA was isolated from frozen artery segments using TRIzol reagent (Invitrogen, Carlsbad, CA) according to the manufacturer's instructions. The concentration and purity of the RNA in each sample were determined using a spectrophotometer at 260 and $280 \mathrm{~nm}$. cDNA was synthesized from $1 \mu \mathrm{g}$ of total-RNA using a PrimeScript RT reagent kit (Takara, Dalian, China). Quantitative real-time RT-PCR was performed using SYBRGreen (Takara Biotechnology) on an Exicycler ${ }^{\mathrm{TM}} 96$ real-time quantitative thermal block (Bioneer, Daejeon, Korea). The PCR primer sequences were designed according to the rat $\mathrm{CNP}$ and

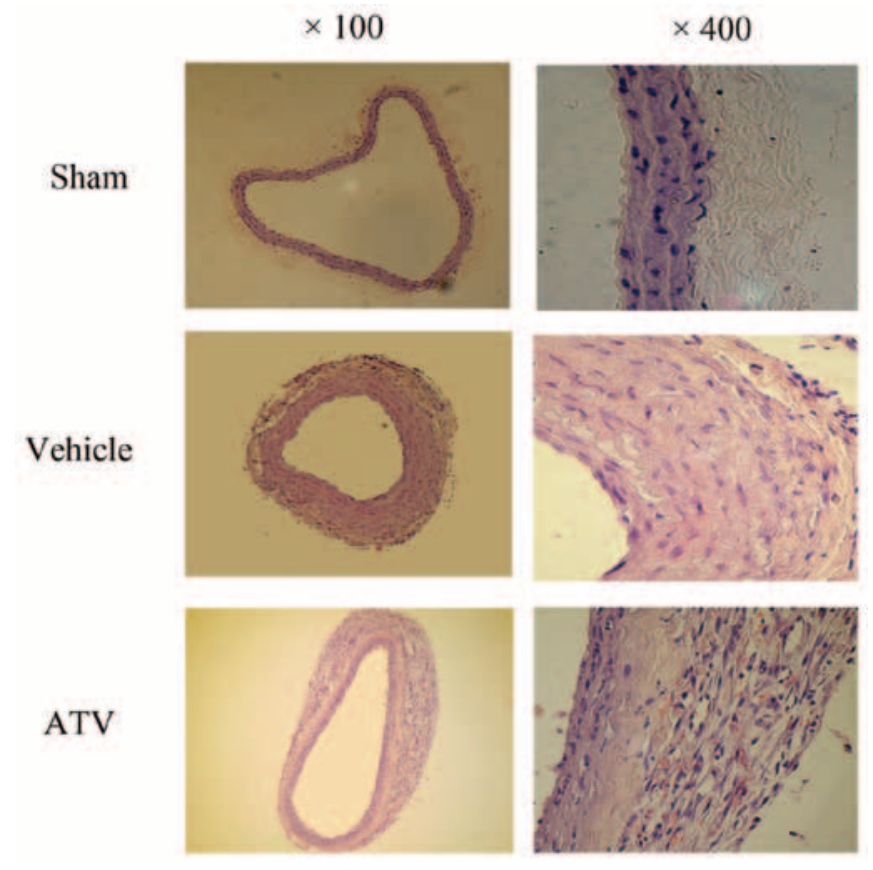

Figure 1. Photomicrographs of paraffin transverse sections of carotid arteries after staining with haematoxylin and eosin (H\&E).

glyceraldehyde-3-phosphate dehydrogenase (GAPDH) gene sequences reported in GenBank and were chemically synthesized: CNP, forward, 5'-CACCATGCACCTCTCCCAGC-3' and reverse, 5'-ATGGAGCCGATCCGGTCCAG-3'; GAPDH, forward, 5'-GCAAGTTCAACGGCACA-3' and reverse, 5'-CATTTGATGTTAGCGGGAT-3'. The specificity of the amplified products was analyzed through dissociation curves generated by the equipment yielding single peaks. GAPDH was used as an internal control to normalize samples. PCR reactions of each sample were conducted in triplicate. Data were analyzed through the comparative threshold cycle (CT) method.

Western blot analysis. The frozen artery segments were lysed in RIPA buffer (Beyotime Institute of Biotechnology, Haimen, China) and the protein concentrations were determined by the BCA assay. Equal amounts of protein were separated by SDS-PAGE and then electrotransferred to PVDF membranes (Millipore, Billerica, MA). The blotted membranes were blocked with $5 \%$ skim milk at $4^{\circ} \mathrm{C}$ overnight, and then incubated with goat anti-cGMP-dependent protein kinase (PKG) I $\alpha$ or anti-PKG I $\beta$ (Santa Cruz Biotechnology, Santa Cruz, CA, USA, 1:1000) at room temperature for $2 \mathrm{~h}$. After incubation with the horseradish peroxidase-conjugated goat anti-rabbit secondary antibody, visualization was performed by an enhanced chemiluminescence kit. Immunoblotting with the anti-actin antibody was used as an internal control to confirm equivalent protein loading.

Statistical analyses. Numerical data are presented as mean \pm SD and were analyzed by the Student's t-test using SPSS 13.0 software (SPSS Inc., Chicago, IL, USA). A P-value $<0.05$ was considered to denote statistical significance. 
Table I. Effect of atorvastatin (ATV) treatment on neointimal information in carotid arteries.

\begin{tabular}{lccr}
\hline Group & Sham & Vehicle & ATV \\
\hline Medial area $\left(\mathrm{mm}^{2}\right)$ & $0.130 \pm 0.008$ & $0.066 \pm 0.002^{\mathrm{a}}$ & $0.060 \pm 0.003^{\mathrm{a}}$ \\
Intimal area $\left(\mathrm{mm}^{2}\right)$ & undefined $^{\mathrm{c}}$ & $0.009 \pm 0.001$ & $0.004 \pm 0.001^{\mathrm{b}}$ \\
I/M ratio & undefined & $0.13 \pm 0.05$ & $0.06 \pm 0.01^{\mathrm{b}}$
\end{tabular}

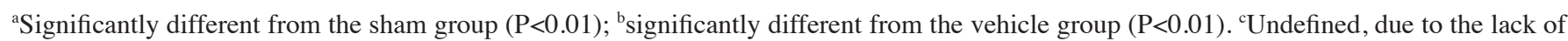
intimal growth. Values are expressed as the mean \pm SD from 8-10 experiments.

A

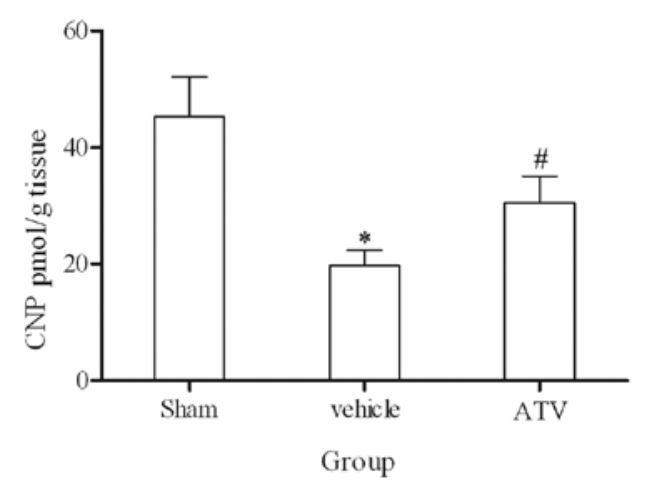

B

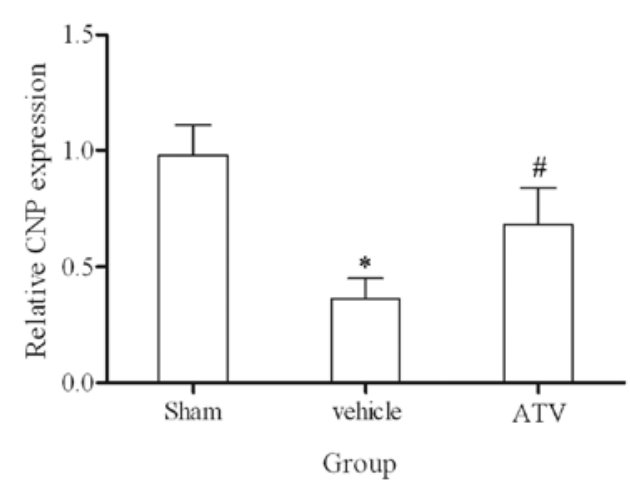

Figure 2. (A) Expression of CNP in carotid arteries as determined by ELISA assays and (B) quantitative real-time $\mathrm{RT}-\mathrm{PCR}$. ${ }^{*} \mathrm{P}<0.05$ v.s. sham group; ${ }^{*} \mathrm{P}<0.05$ v.s. vehicle group.
A

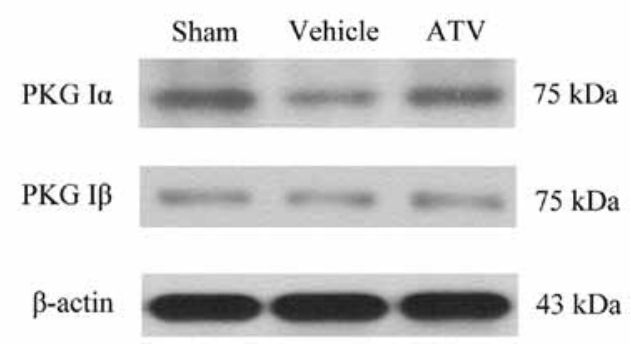

B

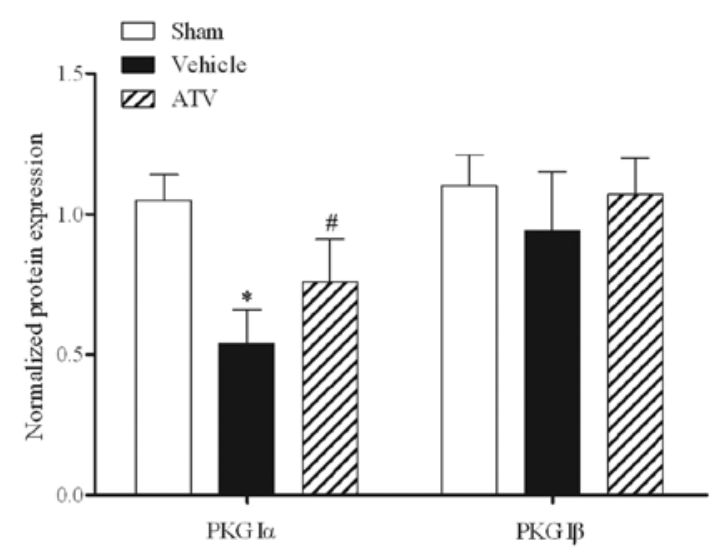

Figure 3. Protein levels of PKG I $\alpha$ and PKG I $\beta$ in carotid arteries. (A) Representative western blotting of three independent and reproducible experiments. (B) Quantitative data were expressed as the intensity ratio of the target genes to GAPDH.

\section{Results}

Effect of ATV on neointima formation induced by perivascular collars. In the present study we investigated the effect of ATV administration on the development of an atheroma-like neointima induced by application of a peri-arterial collar in rats. As shown in Fig. 1 and Table I, the medial area in both the vehicle- and ATV-collared arteries was significantly reduced when compared to the sham segment. In collared arteries, ATV treatment did not affect the medial changes but it significantly suppressed the neointimal growth showing a reduction in both intimal area and IMR when compared to vehicle treatment. The sham segment remained unaffected (Table I). These observations suggest that administration of ATV was effective in preventing the development of a neointima in the atherosclerotic rat model.
Effect of ATV on CNP expression in carotid arteries. To further explore the possible mechanisms by which ATV prevents the formation of a neointima, we examined the concentration and mRNA level of CNP in carotid arteries using ELISA and quantitative real-time RT-PCR, respectively. As shown in Fig. 2A, in comparison to the arteries in the sham group, the concentrations of $\mathrm{CNP}$ were markedly reduced in the vehicle group $(\mathrm{P}<0.05)$. However, ATV treatment significantly attenuated this reduction $(\mathrm{P}<0.05)$. Meanwhile, changes observed by quantitative real-time RT-PCR study were in accordance with the findings in the ELISA assays (Fig. 2B). These findings suggest that ATV suppressed the neointimal growth possibly by elevating CNP expression.

Effect of ATV on the expression of PKG I $\alpha$ and PKG I $\beta$ in carotid arteries. CNP has long been known as a potent 
stimulator of cGMP (25). To provide more evidence for the elevation of CNP in carotid arteries, we further examined the protein expression of two PKGs (PKG I $\alpha$ and I $\beta$ ), the serine/ threonine-specific protein kinase that is activated by cGMP. Compared with the sham group, there was a marked decrease in the PKG I $\alpha$ protein level in the vehicle group. This reduction was significantly reversed by ATV treatment $(\mathrm{P}<0.05$, Fig. $3 \mathrm{~A}$ and $\mathrm{B}$ ). However, no obvious changes in PKG I $\beta$ expression were noted among the three groups (Fig. 3A and B). These results not only confirmed the elevation of CNP by ATV treatment, but also indicate that the inhibition of neointimal growth by ATV may be associated with the activation of PKG I $\alpha$.

\section{Discussion}

The present study demonstrated, for the first time, that ATV treatment in the peri-arterial collar model of atherosclerosis in rats prevented the formation of a neointima, usually noted 7 days after the placement of the peri-arterial collar alone. In addition, ATV treatment increased the expression of CNP and PKG I $\alpha$ in carotid arteries. Therefore, ATV may have a protective role in atherosclerosis and this role may prove beneficial in reducing the remodeling associated with the early stages of atherosclerosis.

Placement of a pericarotid or perifemoral collar has been shown to induce intimal thickening in rabbits, rats and mice (26-28). Previous studies have demonstrated that collar placement causes polymorphonuclear leukocyte infiltration and endothelial injury in the initial step by inflammatory responses, followed by the migration of medial vascular smooth muscle cells to the intima and the proliferation of migrated vascular smooth muscle cells with deposition of extracellular matrix in the neointima. These steps are comparable to those observed in the development of human atherosclerosis (29). Consequently, intimal thickening induced by collar placement may be an adequate model to facilitate understanding of atherosclerosis. In this study, intimal thickening of the rat carotid artery was induced by collar placement. Consistent with previous results, a marked neointima formation was observed in the carotid artery of the vehicle group two weeks after the collar treatment, whereas this neointima was significantly inhibited by the ATV treatment. These findings not only confirmed the successful induction of the atherosclerotic rat model, but also suggest that ATV treatment is capable of preventing the development of a neointima.

The protective effects of ATV and CNP on atherosclerosis have been separately reported in different models. For example, Raval et al recently showed that ATV combined with celecoxib, a cyclooxygenase (COX-2) inhibitor, reduced the extent of atherosclerosis and inflammatory/cell adhesion molecule levels in the apo $\mathrm{E}^{-/}$mouse model (30). In addition, Gaspari et al have demonstrated that local infusion of CNP results in preservation of endothelial function and prevention of neointimal thickening in collar-induced atherosclerotic rabbits (31). Nevertheless, a direct association between ATV and CNP has never been described. In the present study, we found that ATV treatment inhibits the neointima formation in an atherosclerotic rat model, which was accompanied by increases in CNP expression, suggesting that ATV may exert its protective effect by upregulating CNP. Furthermore, previous investigations have clearly demonstrated that the antiinflammatory mechanisms of statins may involve upregulation of transforming growth factor $\beta$ (TGF- $\beta$ ) $(32,33)$. In addition, the expression of CNP is reported to be influenced by several physiological and pathological mediators relevant to the cardiovascular system including upregulation by TGF- $\beta$ and downregulation by oxidized LDL (15). Based on these findings, it therefore seems reasonable to propose that activation of the TGF- $\beta$ signaling pathway may represent one underlying mechanism for the protective effect on atherosclerosis by upregulating the expression of CNP. However, the precise mechanisms by which ATV increases the CNP expression awaits further elucidation.

In conclusion, ATV treatment inhibited the development of a neointima in an atherosclerotic rat model, accompanied by the increase in CNP expression in carotid arteries. Thus, the present data suggest that elevation of CNP represents an additional mechanism by which ATV treatment may prevent the development and progression of atherosclerosis.

\section{References}

1. Ross R: Atherosclerosis - an inflammatory disease. N Engl J Med 340: 115-126, 1999.

2. Hansson GK: Inflammation, atherosclerosis, and coronary artery disease. N Engl J Med 352: 1685-1695, 2005.

3. Rocha VZ and Libby P: Obesity, inflammation, and atherosclerosis. Nat Rev Cardiol 6: 399-409, 2009.

4. Nissen SE, Nicholls SJ, Sipahi I, et al: Effect of very highintensity statin therapy on regression of coronary atherosclerosis: the ASTEROID trial. JAMA 295: 1556-1565, 2006.

5. Randomised trial of cholesterol lowering in 4444 patients with coronary heart disease: the Scandinavian Simvastatin Survival Study (4S). Lancet 344: 1383-1389, 1994.

6. Shepherd J, Cobbe SM, Ford I, et al: Prevention of coronary heart disease with pravastatin in men with hypercholesterolemia. West of Scotland coronary prevention study group. N Engl J Med 333: 1301-1307, 1995.

7. Sacks FM, Pfeffer MA, Moye LA, et al: The effect of pravastatin on coronary events after myocardial infarction in patients with average cholesterol levels. Cholesterol and recurrent events trial investigators. N Engl J Med 335: 1001-1009, 1996.

8. Heart Protection Study Collaborative Group: MRC/BHF heart protection study of cholesterol lowering with simvastatin in 20,536 high-risk individuals: a randomised placebo-controlled trial. Lancet 360: 7-22, 2002.

9. Hebert PR, Gaziano JM, Chan KS and Hennekens CH: Cholesterol lowering with statin drugs, risk of stroke, and total mortality. An overview of randomized trials. JAMA 278: 313-321, 1997.

10. Maron DJ, Fazio S and Linton MF: Current perspectives on statins. Circulation 101: 207-213, 2000.

11. Ridker PM, Danielson E, Fonseca FA, et al: Rosuvastatin to prevent vascular events in men and women with elevated C-reactive protein. N Engl J Med 359: 2195-2207, 2008.

12. Albert MA, Danielson E, Rifai N and Ridker PM: Effect of statin therapy on C-reactive protein levels: the pravastatin inflammation/CRP evaluation (PRINCE): a randomized trial and cohort study. JAMA 286: 64-70, 2001.

13. Kinlay S, Schwartz GG, Olsson AG, et al: High-dose atorvastatin enhances the decline in inflammatory markers in patients with acute coronary syndromes in the MIRACL study. Circulation 108: 1560-1566, 2003.

14. Scotland RS, Ahluwalia A and Hobbs AJ: C-type natriuretic peptide in vascular physiology and disease. Pharmacol Ther 105: 85-93, 2005.

15. Ahluwalia A and Hobbs AJ: Endothelium-derived C-type natriuretic peptide: more than just a hyperpolarizing factor. Trends Pharmacol Sci 26: 162-167, 2005.

16. Murakami S, Nagaya N, Itoh T, et al: C-type natriuretic peptide attenuates bleomycin-induced pulmonary fibrosis in mice. Am J Physiol Lung Cell Mol Physiol 287: L1172-L1177, 2004. 
17. Scotland RS, Cohen M, Foster P, et al: C-type natriuretic peptide inhibits leukocyte recruitment and platelet-leukocyte interactions via suppression of P-selectin expression. Proc Natl Acad Sci USA 102: 14452-14457, 2005.

18. Shinomiya M, Tashiro J, Saito Y, et al: C-type natriuretic peptide inhibits intimal thickening of rabbit carotid artery after balloon catheter injury. Biochem Biophys Res Commun 205: 1051-1056, 1994.

19. Ueno H, Haruno A, Morisaki N, et al: Local expression of C-type natriuretic peptide markedly suppresses neointimal formation in rat injured arteries through an autocrine/paracrine loop. Circulation 96: 2272-2279, 1997.

20. Doi K, Ikeda T, Itoh H, et al: C-type natriuretic peptide induces redifferentiation of vascular smooth muscle cells with accelerated reendothelialization. Arterioscler Thromb Vasc Biol 21: 930-936, 2001

21. Casco VH, Veinot JP, Kuroski de Bold ML, Masters RG, Stevenson MM and de Bold AJ: Natriuretic peptide system gene expression in human coronary arteries. J Histochem Cytochem 50: 799-809, 2002.

22. Barrios V, Amabile N, Paganelli F, et al: Lipid-altering efficacy of switching from atorvastatin $10 \mathrm{mg} /$ day to ezetimibe/simvastatin $10 / 20 \mathrm{mg} /$ day compared to doubling the dose of atorvastatin in hypercholesterolaemic patients with atherosclerosis or coronary heart disease. Int J Clin Pract 59: 1377-1386, 2005.

23. Daenen S, Muskiet FA, Marrink J and Halie MR: Aggressive chemotherapy for acute leukaemia frequently causes intestinal protein leakage. Eur J Cancer 27: 552-556, 1991.

24. Nohria A, Prsic A, Liu PY, et al: Statins inhibit Rho kinase activity in patients with atherosclerosis. Atherosclerosis 205 517-521, 2009

25. Yeung VT, Ho SK, Cockram CS, Lee CM and Nicholls MG: C-type natriuretic peptide is a potent stimulator of cyclic GMP production in cultured mouse astrocytes. J Neurochem 59: 762-764, 1992
26. Booth RF, Martin JF, Honey AC, Hassall DG, Beesley JE and Moncada S: Rapid development of atherosclerotic lesions in the rabbit carotid artery induced by perivascular manipulation. Atherosclerosis 76: 257-268, 1989.

27. Akishita M, Ouchi Y, Miyoshi H, et al: Estrogen inhibits cuffinduced intimal thickening of rat femoral artery: effects on migration and proliferation of vascular smooth muscle cells. Atherosclerosis 130: 1-10, 1997.

28. Moroi M, Zhang L, Yasuda T, et al: Interaction of genetic deficiency of endothelial nitric oxide, gender, and pregnancy in vascular response to injury in mice. J Clin Invest 101: 1225-1232, 1998.

29. Ross R: The pathogenesis of atherosclerosis: a perspective for the 1990s. Nature 362: 801-809, 1993.

30. Raval M,FrankPG,Laury-Kleintop L, Yan G and Lanza-Jacoby S: Celecoxib combined with atorvastatin prevents progression of atherosclerosis. J Surg Res 163: e113-e122, 2010.

31. Gaspari TA, Barber MN, Woods RL and Dusting GJ: Type-C natriuretic peptide prevents development of experimental atherosclerosis in rabbits. Clin Exp Pharmacol Physiol 27: 653-655, 2000.

32. Porreca E, Di Febbo C, Baccante G, Di Nisio M and Cuccurullo F: Increased transforming growth factor-beta(1) circulating levels and production in human monocytes after 3-hydroxy-3-methylglutaryl-coenzyme a reductase inhibition with pravastatin. J Am Coll Cardiol 39: 1752-1757, 2002.

33. Rodriguez-Vita J, Sanchez-Galan E, Santamaria B, et al: Essential role of TGF-beta/Smad pathway on statin-dependent vascular smooth muscle cell regulation. PLoS One 3: e3959, 2008. 